\title{
RB scheduling and power allocation in Carrier Aggregation Systems
}

\author{
Jia $\mathrm{Yu}^{1,2,}$, Shinsuke Konaka ${ }^{1, b}$, Masatake Akutagawa, ${ }^{1, c}, Y_{i}$ Yang $^{1,2, d}$, \\ Ye Wang ${ }^{2, e}$ and Qinyu Zhang ${ }^{2, f}$ \\ ${ }^{1}$ Graduate School of Advanced Technology and Science, Tokushima University, Tokushima, Japan \\ ${ }^{2}$ Communication Engineering Research Center (CERC), Shenzhen Graduate School, Harbin \\ Institute of Technology, Shenzhen, Guangdong, P. R. China \\ ayujia_hitsz@hotmail.com, ${ }^{b}$ konaka@ee.tokushima-u.ac.jp, ’makutaga@ee.tokushima-u.ac.jp, \\ dwaiwaisnk25@hotmail.com, e'wangye.hitsz@gmail.com, 'zqy@hit.edu.cn
}

Keywords: Radio Resource Management, Optimization, Carrier Aggregation, LTE-Advanced.

Abstract. Carrier aggregation (CA) technique is introduced by 3GPP to support higher data rate transmissions over up to $100 \mathrm{MHz}$ frequency bandwidths in LTE-Advanced (LTE-A) systems. However, the use of CA brings new challenges to radio resource management (RRM). In this paper, we propose RRM algorithms, involving resource block (RB) scheduling and power allocation.

\section{Introduction}

Carrier Aggregation (CA) technique is introduced into LTE-Advanced (LTE-A) systems [1] [2], as an efficacious method to significantly improve spectrum efficiency of mobile communication systems. A user equipment (UE) supporting CA technique is supposed to be equipped with multiple independent radio frequency chains (RFCs) and can be simultaneously scheduled on multiple component carriers (CCs). Nevertheless, the application of the CA technique brings new challenges, especially in terms of radio resource management (RRM) of LTE-A systems. Computation complexity of RRM is greatly intensified when resource blocks (RBs), power and CC are jointly considered.

There have been a few literatures on resource allocation of CA systems [3] [4] [5]. These works are under a similar study framework that first decomposes the complex allocation problem into several subproblems, and then solves these sub-problems step by step. Although the decomposed framework may not get the optimal solution comparing with jointly allocation, it can significantly reduce.

Given the solution of CC-allocation, RB-allocation can be further grouped into two categories: Independent scheduling in each CC (IC) and Across-CC scheduling (AC). IC scheme refers to allocating $\mathrm{RBs}$ in each $\mathrm{CC}$ without considering others. The advantage of IC scheme is the compatibility with existing RB-allocation algorithms in LTE system. However, IC scheme is obviously a suboptimal solution. On the other hand, AC scheme takes all CCs into consideration when allocating RBs, and it hereby can achieve an overall better resource allocation than that of IC scheme. The main drawback of the $\mathrm{AC}$ scheme lies in the higher complexity. In [3], the authors proposed a quasi-AC scheduling algorithm that upgrades from IC scheduling by taking past UEs' throughput over all aggregated CCs into account. In [4], a generalized AC scheduling was proposed, which introduces two fairness weighting factors, and proves that the proposed AC scheduling can offer higher utility than that of IC scheduling. In [6], the authors proposed a maximum system utility (MSU) scheduling algorithm, by which the best CC is allocated to one narrow-band UE until the total utility cannot grow anymore.

However, most of the aforementioned algorithms are inspired by the idea of the greedy algorithm to maximize certain utility function in each allocation, so these methods often achieve the sub-optimal solution and there is margin to improve the quality of the solution. To solve the RB-allocation efficiently, in this paper, we investigate the problem under the given $\mathrm{CC}$-allocation, and design a modified greedy algorithm based on a RB exchange strategy. Finally, to further balance the tradeoff between fairness and sum capacity, under the condition of the given CC-allocation and the RB-allocation results before, we also investigate the Power-allocation by using particle swarm optimization (PSO) algorithm, a kind of heuristic method, to make this work complete. 


\section{System Model}

We consider a LTE-A system comprising $M$ CCs and $K$ UE. The CC $m$ consists of $N_{m}$ OFDM RBs, and each UE is equipped with $S$ RFCs. Denote RFC index of UE $k$ by $\psi_{k, s}^{m} \in\{0,1\}$, where $\psi_{k, s}^{m}=1$ means RFC $s$ of UE $k$ is allowed to transmit data over CC $m$, and means $\psi_{k, s}^{m}=0$ otherwise. Similarly, denote RB index by $\rho_{k}^{m, n} \in\{0,1\}$, where $\rho_{k}^{m, n}=1$ means RB $n$ of CC $m$ is allocated to UE $k$. Let $p^{m, n}$ denotes the transmit power on RB $n$ of CC $m$, then the data rate of RFC $s$ of UE $k$ on CC $m$ can be expressed as

$$
R_{k, s}^{m}=\psi_{k, s}^{m}\left(\sum_{n=1}^{N_{m}} \rho_{k}^{m, n} W \log _{2}\left(1+\frac{p^{m, n}\left(h_{k, s}^{m, n}\right)^{2}}{\Gamma_{k} N_{0} W}\right)\right),
$$

where $N_{0}$ is the two sided spectral density of additive white Gaussian noise (AWGN); $W$ is the bandwidth of each $\mathrm{RB}, \Gamma_{k}=\frac{1}{3} Q^{-1}\left(\frac{\mathrm{BER}_{k}}{4}\right)^{2}$ is the signal-noise ratio (SNR) gap due to modulation [8], and $\mathrm{BER}_{k}$ is the requirement of bit error rate (BER) for UEs when $M$-QAM is adopted; $h_{k, s}^{m, n}$ is the channel gain for RFC $s$ of UE $k$ on RB $n$ of CC $m$ due to pass loss, shadowing and fading. For simplification, we assume that RFCs are independent and identical for a specific UE, and therefore $h_{k, s}^{m, n}=h_{k}^{m, n}$ and $\psi_{k, s}^{m}=\psi_{k}^{m}, \forall s$. Then the sum capacity of UE $k$ can be expressed as

$$
R_{k}=\sum_{m=1}^{M} R_{k}^{m}=\sum_{m=1}^{M} \sum_{s=1}^{S} R_{k, s}^{m}
$$

Fairness is an important performance metric in multiuser wireless communication systems [9]. By exploiting user fairness, we can explicitly control the capacity ratios among UEs, and ensure that each UE, especially for cell-edge UEs, achieves the expected data rate. In this paper, we adopt Jain's index to evaluate fairness the same as in [9],

$$
\Phi:=\frac{\left(\sum_{k=1}^{K} R_{k}\right)^{2}}{K \sum_{k=1}^{K} R_{k}^{2}}
$$

The maximum value of $\Phi=1$ will be achieved when $R_{1}=R_{k}, \forall k$. In order to make sure that every UE can be scheduled in each allocation, we define a non-continuous point $\Phi=0$, if there is a UE $k$ whose $R_{k}=0$.

The goal of this paper is to find the optimal resource allocation to maximize system sum capacity while guaranteeing fairness among UEs. Mathematically, the problem can be formulated as a constrained optimization problem as,

$$
\begin{array}{ll} 
& (\boldsymbol{\psi}, \boldsymbol{\rho}, \mathbf{p}) \in \arg \max \sum_{k=1}^{K} R_{k} \\
\text { s.t. } \quad \mathrm{C} 1: \quad \sum_{m=1}^{M} \psi_{k}^{m} \leq S, \psi_{k}^{m} \in\{0,1\}, \forall k \\
\mathrm{C} 2: \quad \sum_{k=1}^{K} \sum_{n=1}^{N_{m}} \rho_{k}^{m, n}=1, \rho_{k}^{m, n} \in\{0,1\}, \forall m \\
\mathrm{C} 3: \quad \sum_{n=1}^{N_{m}} p^{m, n} \leq P_{\text {total }}^{m}, p^{m, n} \in\left[0, P_{\text {total }}^{m}\right], \forall m, n \\
\mathrm{C} 4: \quad \quad \Phi=1
\end{array}
$$


where $\psi:=\left[\psi_{k}^{m}\right]_{K \times M}$ and $\boldsymbol{\rho}:=\left[\rho_{k}^{m, n}\right]_{K \times M \times\left(\max N_{m}\right)}$ indicate the assignments of $\mathrm{CC}$ and $\mathrm{RB}$, respectively. The constraint $\mathrm{C} 1$ implies that a UE can transmit data over $S$ CCs at most; C2 indicates that each RB can only be assigned to one UE at a time; $\mathrm{C} 3$ means the total power of all RBs should be positive but less than the power limitation $P_{\text {total }}^{m}$ of CC $m, \forall m$; and $\mathrm{C} 4$ is the fairness constraint.

The initial problem in Eq. 4 is a typical mix-integer nonlinear programming (MINP) problem, which is believed difficult to be solved in polynomial time. Here, we adopt a skill of transferring objective function that combines the constraint $\mathrm{C} 4$ into the original objection function. The rationale behind this transformation follows the proposition described below.

Proposition 1: Under the constraint condition of $\Phi=1$, if $(\boldsymbol{\psi}, \boldsymbol{\rho}, \mathbf{p}) \in \arg \max \sum_{k=1}^{K} R_{k}$, then $(\boldsymbol{\psi}, \boldsymbol{\rho}, \mathbf{p}) \in \arg \max \min R_{k}$.

Proof: Suppose there is a solution $\left(\boldsymbol{\psi}^{\prime}, \boldsymbol{\rho} \boldsymbol{\rho}^{\prime}, \mathbf{p}^{\prime}\right)$ that satisfies with $\min R_{k}^{\prime}>\min R_{k}$. According to definition in Eq. 3 and constraint $\Phi=1, \sum_{k=1}^{K} R_{k}^{\prime}=K \min R_{k}^{\prime}>\sum_{k=1}^{K} R_{k}=K \min R_{k}$, which is contradictory with $(\boldsymbol{\psi}, \boldsymbol{\rho}, \mathbf{p}) \in \arg \max \sum_{k=1}^{K} R_{k}$, thus $\left(\boldsymbol{\psi}^{\prime}, \boldsymbol{\rho}^{\prime}, \mathbf{p}^{\prime}\right)$ does not exist.

According to Proposition 1, the initial problem can be transformed into the following one,

(P) $\boldsymbol{\psi} \in \arg \max \min R_{k}(\boldsymbol{\rho}, \mathbf{p})$

s.t. $\quad \mathrm{C} 1: \quad \sum_{m=1}^{M} \psi_{k}^{m} \leq S, \Psi_{k}^{m} \in\{0,1\}, \forall k$

$\mathrm{C} 2: \quad \sum_{k=1}^{K} \sum_{n=1}^{N_{m}} \rho_{k}^{m, n}=1, \rho_{k}^{m, n} \in\{0,1\}, \forall m$

C3: $\quad \sum_{n=1}^{N_{m}} p^{m, n} \leq P_{\text {total }}^{m}, p^{m, n} \in\left[0, P_{\text {total }}^{m}\right], \forall m, n$

To simplify problem ( $\mathrm{P}$ ), we adopt a decomposed framework that decomposes the problem into three suboptimal problems, CC-allocation, RB-allocation and power-allocation. In this paper, we assume that $\mathrm{CC}$ allocation results are given. We solve $\mathrm{RB}$-allocation under the given $\mathrm{CC}$-allocation results and approximate power-allocation, the problem of power-allocation can be derived naturally. The decomposed suboptimal problems can be written:
(P 1) $\boldsymbol{\psi} \in \arg \max \min R_{k}(\boldsymbol{\rho}, \mathbf{p})$
s.t. $\quad \mathrm{C} 1 \quad \sum_{m=1}^{M} \psi_{k}^{m} \leq S, \psi_{k}^{m} \in\{0,1\}, \forall k$
(P 2) $\boldsymbol{\rho} \in \arg \max \min R_{k}(\boldsymbol{\psi}, \mathbf{p})$
s.t. $\quad \mathrm{C} 2 \quad \sum_{k=1}^{K} \sum_{n=1}^{N_{m}} \rho_{k}^{m, n}=1, \rho_{k}^{m, n} \in\{0,1\}, \forall m$
(P 3) $\mathbf{p} \in \arg \max \min R_{k}(\boldsymbol{\psi}, \boldsymbol{\rho})$
s.t. $\quad \mathrm{C} 3 \quad \sum_{n=1}^{N_{m}} p^{m, n} \leq P_{\text {total }}^{m}, p^{m, n} \in\left[0, P_{\text {total }}^{m}\right], \forall m, n$
(P 1) can be solved by the algorithm proposed in our previous work [7]. In the following sections, we will present the respective solution to (P 2 ) and (P 3 ). 


\section{Resource Block Allocation}

In this section, we investigate the $\mathrm{RB}$-allocation problem under the given $\mathrm{CC}$-allocation results and estimated power-allocation. We assume that power is equal distributed on all RBs of the same CC. define the virtual power $P:=P_{\text {total }}^{1} / N_{1}$, and then we can get

$$
p^{m, n}=p^{m,-}=\frac{P_{\text {total }}^{m}}{N_{m}}=\frac{P_{\text {total }}^{m} N_{1}}{P_{\text {total }}^{1} N_{m}} P, \forall m, n \text {. }
$$

We also define $H_{k}^{m, n}=\frac{\left|h_{k}^{m, n}\right|^{2} p^{m,-}}{\Gamma N_{0} W P}$ as the virtual channel fading for UE $k$ over RB $n$ of CC $m$.

To solve the RB allocation problem, we use a greedy RB-allocation algorithm in [9] and [10] at the first place. Then, we present a RB exchange based greedy algorithm for RB allocation using the ratio of channel fading between two UEs in the same CC. This algorithm is mainly inspired by our previous work [11] on subcarrier allocation problem of OFDMA system.

Greedy Resource Block Allocation Algorithm (MG-RBA). We first discuss the RB-allocation problem based on [9] and [10]. The intuition behind this greedy algorithm is to maximize the sum capacity by taking advantage of multiuser diversity as possible, while maintaining a coarse proportional fairness. Based on the greedy allocation, we design a modified greedy RB-allocation algorithm (MG-RBA) to make it suited to our problem.

The proposed MG-RBA algorithm consists of two stages. For the first stage, each UE selects one $\mathrm{RB}$ on the allocated CCs in sequence. After that, the UE with minimal capacity has the priority to select the best one of rest RBs. This procedure repeats until all RBs are assigned to the corresponding UEs.

RB Exchange based Resource Block Algorithm (RE-RBA). Based on the proposed MG-RBA algorithm, we further proposed an RB exchange based algorithm (RE-RBA) to further balance the tradeoff between sum capacity and proportional fairness. Specifically, for given solution $\boldsymbol{\rho}$ of MG-RBA, we can obtain the capacity $R_{k}, \forall_{k}$ under the assumption of equal power distribution. After that, the algorithm finds the minimal capacity UE $k_{\min }$ and its maximal capacity CC $m_{\max }$. Then, we adopt a conservative strategy to exchange RB from some UE to UE $k_{\min }$ within CC $m$. According to [11], the candidate $\mathrm{RB} n^{*}$ transferring to $k_{\min }$ with lowest loss of sum capacity has to satisfy condition $\max _{n}\left\{H_{k_{\min }}^{m_{\max }, n} / H_{k^{\prime}}^{m_{\max }, n}\right\}$, where $k^{\prime}\left(k^{\prime} \neq k_{\min }\right)$ is the current owner of RB $n^{*}$ in CC $m_{\max }$. If such an RB exist, set $\rho_{k_{\operatorname{mix}}}^{m_{\max }, n^{*}}=1, \rho_{k^{\prime}}^{m_{\max }, n^{*}}=0$, and recalculate capacity $R_{k_{\min }}=R_{k_{\min }}+W \log _{2}\left(1+P H_{k_{\min }}^{m_{\max }, n^{*}}\right)$, and $R_{k^{\prime}}=R_{k^{\prime}}-W \log _{2}\left(1+P H_{k^{\prime}}^{m_{\max }, n^{*}}\right)$. This process is repeated until we cannot find out such RB that can improve the capacity of certain $R_{k_{\min }}$, and the algorithm stops. The detailed implementation of RE-RBA is summarized in Algorithm 1. 


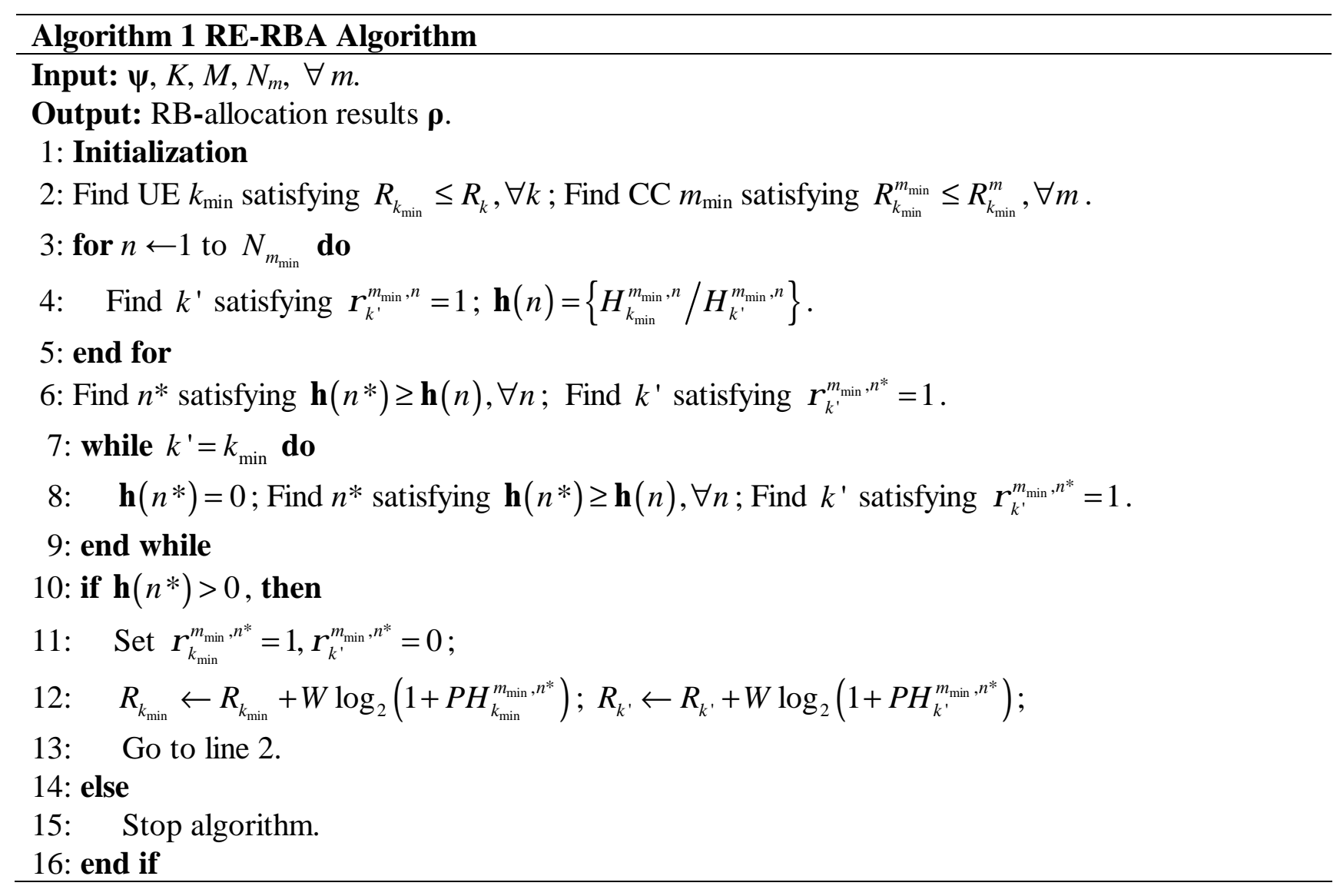

\section{Power Allocation.}

After RB-allocation, we can further improve the objective by distributing power on each RB. Substituting the obtained $\rho$ with the given $\psi$ into problem (P 3), the power allocation can be seen as a constrained continuous variable optimization.

In this section, we propose a heuristic method based on the particle swarm optimization (PSO) algorithm. In contrast to other heuristic methods, PSO has better global searching capability at the beginning of the run and a local searching capability near the end of the run thanks to the connections among particles [12]. The standard PSO algorithm consists of the following steps. (1) Construct the particle to map the solution of interest problem, (2) Create the topology of particle swarm (particles' position), (3) Calculate fitness value for each particle and find out the best particle, (4) Updating particles' position, (5) Repeat step (3)-(4) until the stopping criterion is satisfied. Alone with the standard PSO algorithm, the procedure of the proposed CE based power allocation (PSO-PA) is explained as follows.

Step 1(Particle Construction): Assume there are $Z^{\text {pso }}$ particles. We denote vector

$$
\text { particle }_{z}=\left\{\text { particle }_{z}^{m}\right\}, m=\{1, \ldots, M\}
$$

as the $z$ th particle, where $\operatorname{particle}_{z}^{m}=\left\{\operatorname{particle}_{z}^{m}(n)\right\}, n=1, \ldots, N_{m}$ is an $N_{m}$-dimension sub-vector that represents the power allocation in CC $m$, and each element $\operatorname{particle}_{z}^{m}(n)$ in the vector is a power value. The particular particle structure is illustrated in Fig. 1. Accordingly, we denote the vector velocity with the same structure as the velocity of the $z$ th particle, and initialize it as the following expression:

$$
\operatorname{velocity}_{z}^{m}(n)=p^{m,-}(\omega-0.5), \forall m, n, z
$$

where $\omega$ follows standard uniform distribution in $[0,1]$. 


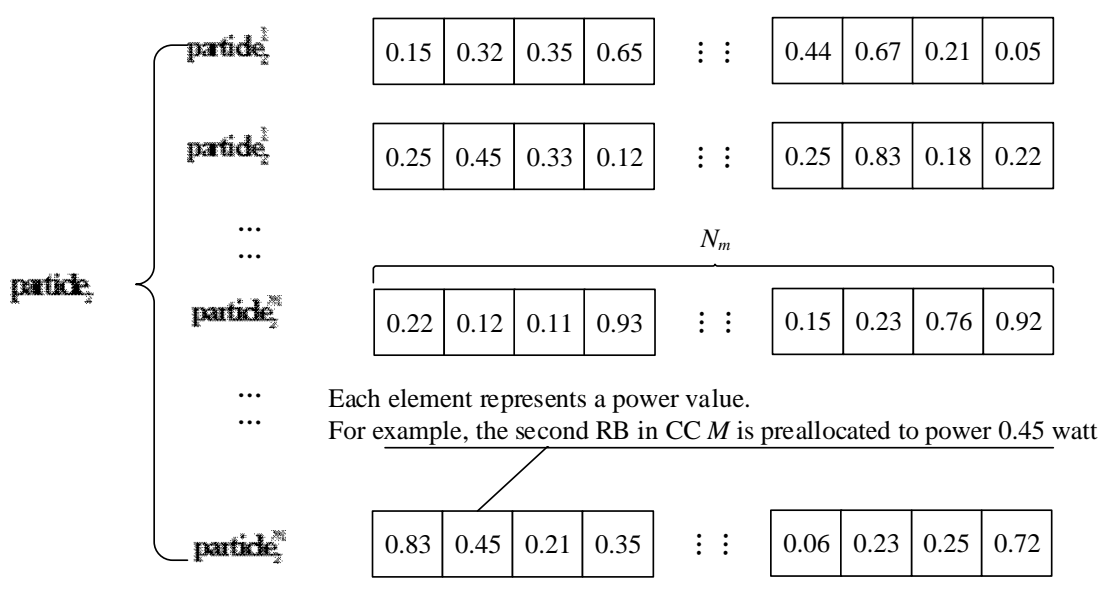

Fig. 1 An illustration of particle structure.

Step 2 (Topology Creation): The target of topology creation is to initialize the position particles (the value of each element in particle $\mathbf{e}_{z}$ ) and its velocity. We randomize all particles around equation power distribution by the following expression:

$$
\operatorname{particle}_{z}^{m}(n)=p^{m,-}+p^{m,-}(\omega-0.5), \forall m, n, z
$$

Step 3(Particle Adapting): The process of particle adapting is to evaluate the current particle position and pick out the best one for particle updating and the final solution. Each particle is comparable based on a fitness value that is related to the objective function and the constrain conditions. As to the problem ( $\mathrm{P} 3$ ), we define the fitness function of each particle using the penalty function skill [13]:

$$
F_{z}=\min _{k} R_{k}\left(\boldsymbol{\psi}, \boldsymbol{\rho}, \text { particle }_{z}\right) \times \lambda_{z}
$$

The fitness function is composed by two parts, where $\left.\min _{k} R_{k}(\boldsymbol{\psi}, \boldsymbol{\rho}, \mathbf{p a r t i c l e})_{z}\right)$ indicates the minimal capacity of all UEs according to $\psi, \rho$ and the current position of particle $z$, and $\lambda$ is a penalty function to ensure the constraint $\mathrm{C} 3$ can be satisfied. The expression of $\lambda$ is

$$
\lambda_{z}=1-\sqrt{\sum_{m=1}^{M}\left(\sum_{n=1}^{N_{m}} \operatorname{paticle~}_{z}^{m}(n)-P_{\text {total }}^{m}\right)^{2}}
$$

Besides, we denote $F^{\text {best }}$ and Gparticle ${ }^{\text {best }}$ as the known highest fitness value and the corresponding

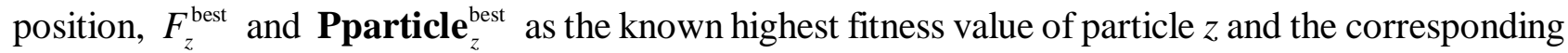
position, respectively.

Step 4 (Particle Updating): After adapting, we should update the particle position and its velocity to approach the excepted optimal solution. According to the suggestion in [14], the particles are manipulated according to the following equations:

$$
\begin{aligned}
& \operatorname{velocity~}_{z}^{m}=\chi\left(\text { velocity }_{z}^{m}+c_{1} \omega_{1} \text { Pparticle }_{z}^{\text {best }}-\text { particle }_{z}^{m}\right)+c_{2} \omega_{2}\left(\text { Gparticle }^{\text {best }}-\text { particle }_{z}^{m}\right) \\
& \text { particle }_{z}^{m}=\text { particle }_{z}^{m}+\text { velocity }_{z}^{m},
\end{aligned}
$$

where $\chi$ is inertia coefficient in PSO algorithm and the variables $\omega_{1}$ and $\omega_{2}$ are random variable with uniform distribution in interval $[0,1]$. The relation between $\chi, c_{2}$ and $c_{1}$ can be given as

$$
\chi=\frac{2}{\left|2-\left(c_{1}+c_{2}\right)-\sqrt{\left(c_{1}+c_{2}\right)^{2}-4\left(c_{1}+c_{2}\right)}\right|},
$$


where $c_{1}=c_{2}=2.05$ and $\chi \approx 0.729$. The algorithm will repeat step 3 and 4 until the stopping criterion is reached. In this paper, we use the maximal iteration times $T_{\mathrm{PSO}}$ as the stopping criterion.

\section{Simulation and analyses.}

Parameter setting. The COST-231 Hata model is considered [15] for large-scale path loss as in a LTE-A network. The corresponding parameters are the same as in our previous work [7]. Other important parameters in the considered LTE-A system are list in Table 1.

\section{Table 1 Simulation parameter setting}

\begin{tabular}{c|c}
\hline Parameter & Value \\
\hline Maximal transmission power in CC $1 P_{\text {total }}^{1}$ & 5 [Watt] \\
Maximal transmission power in CC $2 P_{\text {total }}^{2}$ & 10 [Watt] \\
Maximal transmission power in CC $3 P_{\text {total }}^{3}$ & 10 [Watt] \\
Maximal transmission power in CC $4 P_{\text {total }}^{4}$ & 10 [Watt] \\
Power spectral density $N_{0}$ & $10^{-17}$ \\
Bandwidth of each RB $W$ & $180[\mathrm{kHz}]$ \\
Number of UEs $K$ & $16 \sim 32$ \\
Number of RFCs for each UE $S$ & 2 \\
BER requirement $B E R$ & $10^{-3}$ \\
Number of samples in PSO $Z^{\text {PSO }}$ & 100 \\
Maximal number of iterations in PSO $T^{\text {PSO }}$ & 500 \\
\hline
\end{tabular}

RB Allocation. The cross entropy-based greedy algorithm for CC-allocation (CEGA-CCA) proposed in our previous work [7] is implemented in the simulation at the first place. On the basis of the obtained CC-allocation result, we show the convergence of the proposed RE-RBA algorithm in Fig. 2, where the start points is the performance of the MG-RBA algorithm. As shown in Fig. 2, the RE-RBA converges to a stable solution with only several iterations. Furthermore, it is observed that all performance metrics can be improved with the increase of iterations, which indicates the capability of the proposed algorithm to drag the search out of the local extreme.

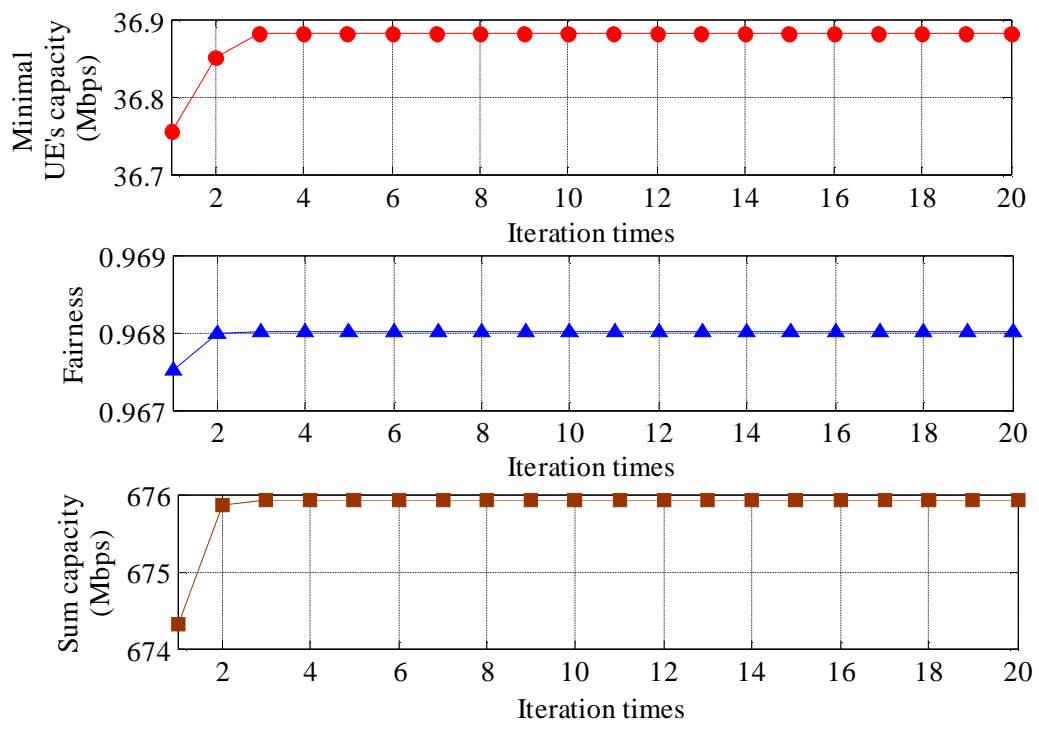

Fig. 2 The convergence of proposed RE-RBA algorithm with different performance metrics

We also present the comparison results of CEGA-CCA without RB allocation, the GA-BRA and RE-BRA in Table 3. In contrast to the result without RB allocation, the proposed GA-RBA algorithm has better performance in terms of minimal UE's capacity and sum capacity, but slightly worse in terms of fairness. This is because the GA-RBA facilitates better use of multi-user diversity. As the enhanced 
method, the proposed RE-RBA is superior to the others in all aspects. Compared to the non-RBA case, the RE-RBA algorithm can obtain $1.7 \%, 0.01 \%$ and $2.2 \%$ improvement in terms of minimal UEs capacity, fairness and sum capacity, respectively.

Table 2 Utility function with optimal channel allocation

\begin{tabular}{c|c|c|c}
\hline Algorithms & Minimal UE's capacity & Fairness & Sum capacity \\
\hline CEGA-CCA & $36.35[\mathrm{MHz}]$ & 0.9679 & $661.45[\mathrm{MHz}]$ \\
CEGA-CCA + GA-RBA & $36.71[\mathrm{MHz}]$ & 0.9675 & $674.32[\mathrm{MHz}]$ \\
CEGA-CCA + RE-RBA & $36.96[\mathrm{MHz}]$ & 0.9680 & $675.93[\mathrm{MHz}]$ \\
\hline CEGA-CCA+PSO-PA & $37.38[\mathrm{MHz}]$ & 0.9782 & $643.09[\mathrm{MHz}]$ \\
CEGA-CCA + GA-RBA + PSO-PA & $37.96[\mathrm{MHz}]$ & 0.9769 & $655.62[\mathrm{MHz}]$ \\
CEGA-CCA + RE-RBA + PSO-PA & $38.08[\mathrm{MHz}]$ & 0.9777 & $657.47[\mathrm{MHz}]$ \\
\hline
\end{tabular}
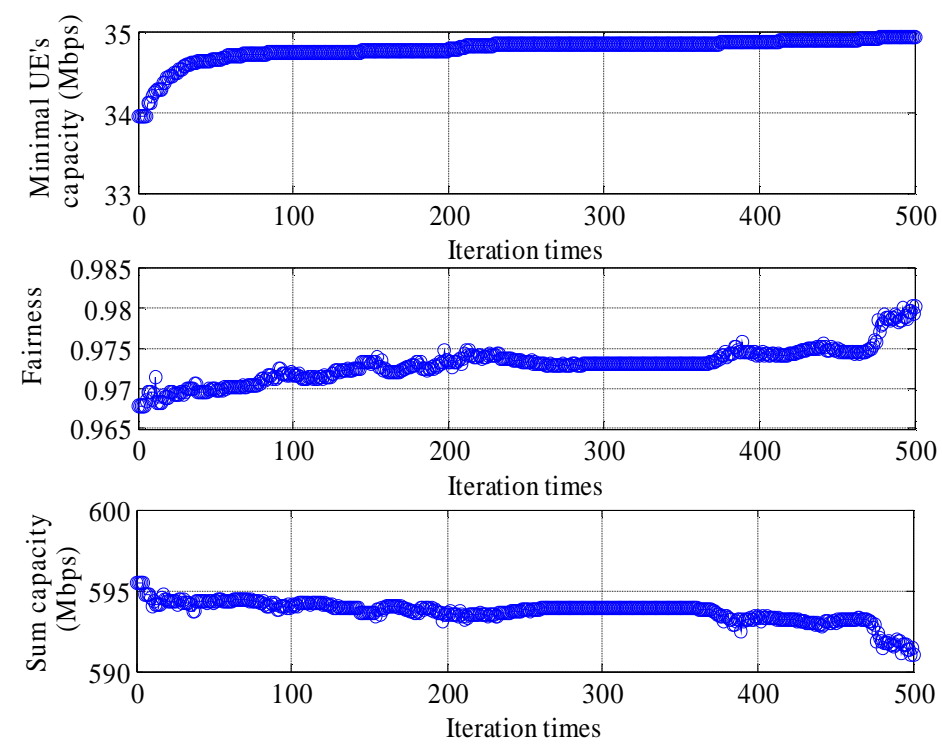

Fig. 3 The convergence of proposed PSO-PA algorithm with different performance metrics

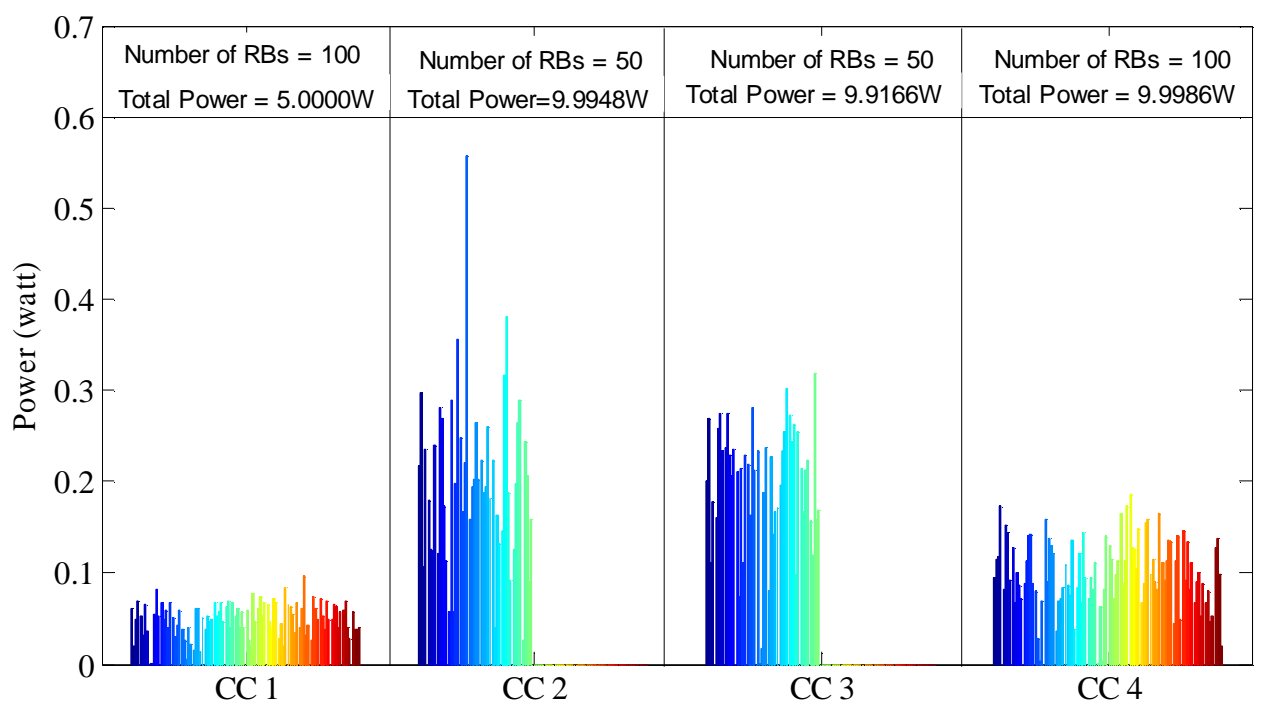

Fig. 4 Power distributions after using the PSO-PA algorithm

Power allocation. Given the results of $\mathrm{CC}$ allocation and $\mathrm{RB}$ allocation, we can further improve the objective of problem (P 3 ) using the proposed PSO-PA algorithm. The performance of the proposed PSO-PA algorithm is shown in Fig. 3. As the increase of iterations, there is a slightly improvement in terms of minimal UE's capacity and fairness, but the sum capacity decreases. This indicates that the power allocation just plays a fine adjustment role in all resource allocation process. We also show the detail power distribution in all RBs. As shown in Fig. 4, we can see that the power limitations in all CCs 
can be satisfied well, which verifies the effectiveness of our designed fitness function in Eq. 14. As shown in Table 2, it is obvious that the system performance in terms of minimal UE's capacity and fairness is improved by the proposed PSO-PA algorithm.

\section{Conclusions}

In this paper, we considered the RRM problem of LTE-A system with CA technique. Jointly resource allocation involving CC-allocation, RB-allocation and power allocation is mathematically formulated. On the base of our previous work, we present a greedy RB-allocation algorithm (GA-RBA), as well as its modified version (RE-RBA). Furthermore, a PSO-based power allocation algorithm is also proposed. Simulation results verify that the proposed algorithms are efficacious to improve system performances.

\section{References}

[1] R. Ratasuk, D. Tolli, and A. Ghosh: Carrier aggregation in lte-advanced, in 2010 IEEE 71st Vehicular Technology Conference (VTC 2010-Spring), p. 1-5.

[2] G. Yuan, X. Zhang, W. Wang, and Y. Yang: Carrier aggregation for lte-advanced mobile communication systems, IEEE Commun. Mag. Vol. 48(2010), p. 88-93.

[3] Y. Wang, K.I. Pedersen, T.B. Sorensen, and P.E. Mogensen: Carrier load balancing and packet scheduling for multi-carrier systems, IEEE Trans on Wireless Commun. Vol. 9(2010), p.1780-1789.

[4] Y. Wang, K. Pedersen, T. Sorensen, and P. Mogensen: Utility maximization in lte-advanced systems with carrier aggregation, in 2011 IEEE 73rd Vehicular Technology Conference (VTC Spring), pp. 1-5.

[5] F. Wu, Y. Mao, S. Leng, and X. Huang: A carrier aggregation based resource allocation scheme for pervasive wireless networks, in 2011 IEEE 9th International Conference on Dependable, Autonomic and Secure Computing (DASC), p. 196-201.

[6] Z. Huang, Y. Ji, and B. Zhao: An efficient resource allocation algorithm with carrier aggregation in lte advanced systems, in 2012 International Conference on Wireless Communications Signal Processing (WCSP), p. s1-6.

[7] Y. Wang, P. Chen, Q. Zhang and X. Lin: Cross entropy based component carrier allocation in LTE-Advanced wireless communication system, 2012 International Conference on Wireless Communications \& Signal Processing (WCSP), p. 1-6.

[8] A. Garcia-Armada: Snr gap approximation for m-psk-based bit loading, IEEE Trans. Wireless Commun. Vol. 5(2006), p. 57-60.

[9] Z. Shen, J. Andrews, and B. Evans: Adaptive resource allocation in multiuser ofdm systems with proportional rate constraints, IEEE Trans. Wireless Commun. Vol. 4(2005), p. 2726-2737.

[10]W. Rhee and J. Cioffi: Increase in capacity of multiuser ofdm system using dynamic subchannel allocation, in 2000 IEEE 51st Vehicular Technology Conference Proceedings (VTC 2000-Spring), Vol. 2, p. 1085-1089.

[11] Y. Wang, Q. Zhang, and N. Zhang: Resource allocation based on subcarrier exchange in multiuser ofdm system, Science China Information Sciences, Vol. 6(2013), p. 1-14, 2013. [Online]. Available: http://dx.doi.org/10.1007/s11432-012-4601-z.

[12]M. Clerc: Particle swarm optimization. John Wiley \& Sons, Vol. 93(2010).

[13]J.M. Yang, Y.P. Chen, J.T. Horng, and C.Y. Kao: Applying family competition to evolution strategies for constrained optimization in Evolutionary Programming VI (1997), p. 201-211.

[14]M. Clerc and J. Kennedy: The particle swarm - explosion, stability, and convergence in a multidimensional complex space, IEEE Trans. Evol. Comput. Vol. 6(2002), p. 58-73.

[15]D. Gomez-Barquero, A. Bria, J. Monserrat, and N. Cardona: Minimal cost planning of $d v b-h$ networks on existing wireless infrastructure, in 2006 IEEE 17th International Symposium on Personal, Indoor and Mobile Radio Communications, p. 1-5. 